\title{
SYNTHESIS AND EVALUATION OF COUMARIN HYBRIDS AS ANTIMYCOBACTERIAL AGENTS
}

\author{
MOHD. ZAHEEN HASSAN ${ }^{1,2 *}$, ABDULRHMAN ALSAYARI ${ }^{1}$, HASNAH OSMAN $^{2}$, \\ MOHAMED ASHRAF ALI $^{3}$, ABDULLATIF BIN MUHSINAH ${ }^{1}$ \\ and MOHAMED JAWED AHSAN ${ }^{4}$,
}

${ }^{1}$ College of Pharmacy, King Khalid University, Abha, KSA 62529

${ }^{2}$ School of Chemical Sciences, Universiti Sains Malaysia, Penang, Malaysia 11800

${ }^{3}$ Institute for Research in Molecular Medicine, Universiti Sains Malaysia, Penang, Malaysia11800

${ }^{4}$ Department of Pharmaceutical Chemistry, Maharishi Arvind College of Pharmacy, Jaipur, India 302023

\begin{abstract}
A series of twelve hybrid coumarin analogs were synthesized and screened through HTS for their antimycobacterial activity against $M t b \mathrm{H} 37 \mathrm{Rv}$. The hybrid molecules were efficiently synthesized by the reactions of 3-(bromoacetyl)coumarin with Biginelli products 2-mercapto-6-oxo-4-aryl-1,6-dihydropyrimidine-5carbonitriles. Of the resulting twelve hybrids, the two compounds 7-(2,4-dichlorophenyl)-5-oxo-3-(2-oxo- $2 \mathrm{H}-$ chromen-3-yl)-5H-thiazolo[3,2-a] pyrimidine-6-carbonitrile (3d) and 7-(4-nitrophenyl)-5-oxo-3-(2-oxo-2Hchromen-3-yl)-5H-thiazolo[3,2-a]pyrimidine-6-carbonitrile (3f) showed excellent antimycobacterial activity against $\mathrm{Mtb}\left(\mathrm{EC}_{50} 3.19 \& 7.91 \mu \mathrm{M}\right.$, respectively) and low cytotoxicity against the VERO cell line $\left(\mathrm{IC}_{50}>62.5\right.$ $\mu \mathrm{g} / \mathrm{mL})$.
\end{abstract}

Keywords: coumarin, thiazolopyrimidine, HTS, TB, cytotoxicity

Tuberculosis (TB) is caused by Mycobacterium tuberculosis $(\mathrm{Mtb})$, which is a very deadly and highly infectious pathogen accountable for millions of deaths per year (1). The recent upsurge in multiple drug-resistant (MDR) and extensively drugresistant (XDR) TB is an alarming concern for TB control programs, as there is no effective treatment available against these resistant strains $(2,3)$. The current treatment options for these resistant $\mathrm{TB}$ strains often require combination drugs which are very expensive and extremely toxic (4). Moreover, anti-TB drug development is progressing very slowly, with only eight new molecules of twelve vaccines are now under clinical trials (Phase I, II and III trials), and the late-stage clinical failure rates are also high (5). Thus, the development of new drugs showing novel mechanisms of action (MOA) against the MDR and XDR TB strains is now a worldwide priority.

Coumarin is an important structural motif in organic and medicinal chemistry, as it is found in numerous natural products and pharmaceutically active compounds. It exhibits diverse biological properties, including antiviral (6), antimicrobial (79), antifungal (10) and anticancer activities (1113). Interest in coumarins has grown due to their antitubercular activity, and several coumarin analogs have been reported as potential anti-TB lead molecules (14). Recently, 7-amino-4-methylcoumarin (NA5) and its acyl derivatives have emerged as potential antitubercular agents. NA5 displayed a remarkable potency against not only H37Rv but also the susceptible as well as the multidrug-resistant clinical isolates superior to isoniazid (15). Moreover, thiazolopyrimidines have also received significant attention as antimycobacterial agents due to their resemblance to purine (16). Encouraged by these observations, we have continued our research on heterocyclic compounds (17, 18). We thought it is worthwhile to synthesize a new series of hybrid derivatives of coumarin containing the thiazolopyrimidine motif, with the hope that the hybridization of the two aforementioned bioactive scaffolds will have a synergistic biological action, resulting in potent antimycobacterial agents (Fig. 1).

\footnotetext{
* Corresponding author: e-mail: drzahin@gmail.com
} 


\section{EXPERIMENTAL}

All the chemicals were purchased from E. Merck (Germany) and Sigma Aldrich. The completion of the reaction was monitored by TLC in a binary solvent system of ethyl acetate and hexane in a $2: 8$ ratio. The compounds were purified by recrystallization with ethylacetate, and the purity of compounds was ascertained by TLC using silica gel G plates (Merck). The spot was developed in an iodine chamber or viewed under a UV lamp. Melting points were determined in an open capillary using a melting point apparatus; these are uncorrected. The magnetic resonance $\left({ }^{1} \mathrm{H}\right.$ and ${ }^{13} \mathrm{C}$ NMR) spectra were recorded on a Brucker $300 \mathrm{MHz}$ instrument in DMSO- $d_{6}$ and $\mathrm{CDCl}_{3}$ using TMS as an internal standard. The infrared spectra of compounds were recorded in $\mathrm{KBr}$ on a JASCO FT-IR instrument.

\section{Synthetic procedures}

\section{2-Mercapto-6-oxo-4-aryl-1,6-dihydropyrimidine-} 5-carbonitriles (1a-l)

These compounds were prepared by the method reported by Kambe et al., 1979, and their physical constants were matched (19).

\section{3-(2-Bromoacetyl)-2H-chromen-2-one (2)}

This compound was prepared using the method reported by Razi et al., 2015, and its physical constant was matched (20).
5-Oxo-3-(2-oxo-2H-chromen-3-yl)-7-aryl-5H-thiazolo[3,2-a]pyrimidine-6-carbonitriles (3a-l)

An equimolar mixture of Biginelli reaction product (1a-l) (2 mmol), and 3-(2-bromoacetyl)2 Hchromen-2-one (2) (0.53 g, 2 mmol) dissolved in ethanol $(20 \mathrm{~mL})$ were refluxed for 5-6 h. After completion of the reaction, as evidenced by TLC, the excess solvent was distilled off and the precipitate thus obtained was filtered, suspended in water and neutralized by aqueous sodium carbonate solution to obtain a free base (3a-l). Finally, the product was filtered, washed with water, dried and recrystallized with ethyl acetate.

5-Oxo-3-(2-oxo-2H-chromen-3-yl)-7-phenyl-5Hthiazolo[3,2-a]pyrimidine-6-carbonitrile (3a)

Yellow solid; m.p. $134-136^{\circ} \mathrm{C}$; yield $80 \%$; IR $(\mathrm{KBr}) \mathrm{v}_{\max }: 2230,1676,1720 \mathrm{~cm}^{-1} ;{ }^{1} \mathrm{H} \mathrm{NMR}\left(\mathrm{CDCl}_{3}\right.$, $300 \mathrm{MHz}) \delta$ ppm: 6.05 (s, 1H, ArH thiazolo $), 6.87$ (d, $2 \mathrm{H}, J=9.0 \mathrm{~Hz}, \mathrm{ArH}), 7.14$ (s, 1H, ArH), 7.20 (d, $2 \mathrm{H}, J=8.7 \mathrm{~Hz}, \mathrm{ArH}), 7.32-7.76(\mathrm{~m}, 5 \mathrm{H}, \mathrm{ArH}) ;{ }^{13} \mathrm{C}$ NMR $\left(\mathrm{CDCl}_{3}, 75 \mathrm{MHz}\right) \delta \mathrm{ppm}: 92.6,101.6,116.2$, $116.8,118.1,122.2,123.4,124.3,125.1,126.9$, $127.3,127.8,132.6,146.3,152.1,157.2,158.1$, 159.7, 160.1, 168.6; MS (ESI): $\mathrm{m} / z 398[\mathrm{M}+1]$; Analysis: calcd for $\mathrm{C}_{22} \mathrm{H}_{11} \mathrm{~N}_{3} \mathrm{O}_{3} \mathrm{~S}$ : C, 66.94 (66.80); H, 2.79 (2.81); N, 10.57 (10.47).

7-(4-Chlorophenyl)-5-oxo-3-(2-oxo-2H-chromen3-yl)-5H-thiazolo[3,2-a]pyrimidine-6-carbonitrile $(3 b)$

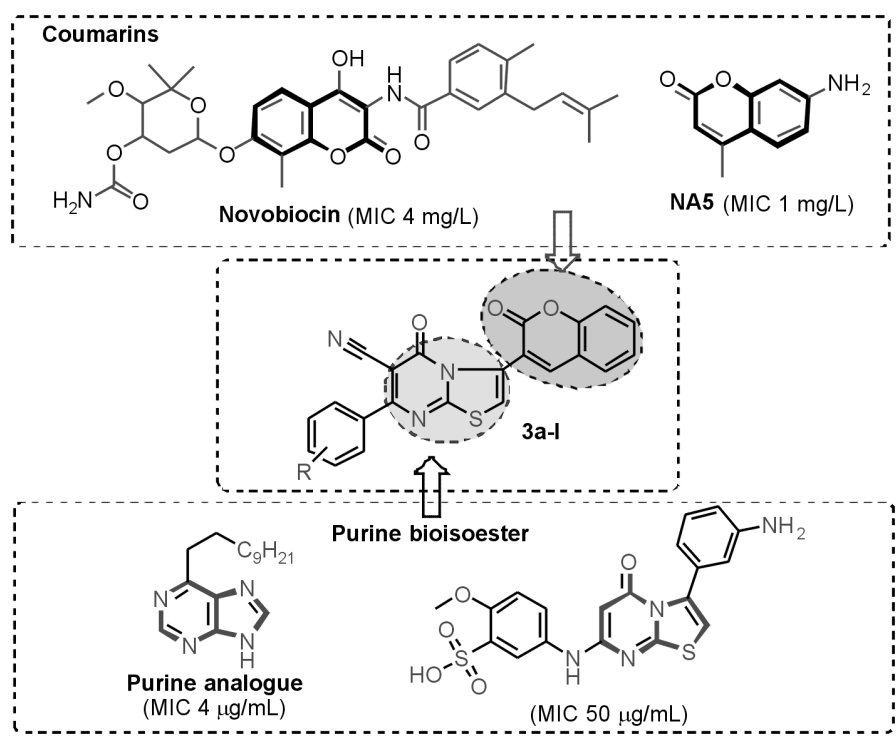

Figure1. Representative structures of anti-tubercular agents which inspired the design of the required scaffolds. 
Pale yellow solid; m.p. $150-152^{\circ} \mathrm{C}$; yield $80 \%$; IR (KBr) $v_{\max }$ : 2222, 1678, $1732 \mathrm{~cm}^{-1}$; ${ }^{1} \mathrm{H}$ NMR $\left(\mathrm{DMSO}_{6}, 300 \mathrm{MHz}\right) \delta \mathrm{ppm}: 6.02(\mathrm{~s}, 1 \mathrm{H}$, $\left.\mathrm{ArH}_{\text {thiazolo }}\right), 6.69-694(\mathrm{~m}, 4 \mathrm{H}, \mathrm{ArH}), 7.26(\mathrm{~s}, 1 \mathrm{H}$, ArH), 7.52 (d, 2H, $J=7.2 \mathrm{~Hz}, \operatorname{ArH}), 7.73$ (d, 2H, $J$ $=7.2 \mathrm{~Hz}, \mathrm{ArH}) ;{ }^{13} \mathrm{C}$ NMR $\left(\mathrm{DMSO}-d_{6,} 75 \mathrm{MHz}\right) \delta$ ppm: 93.1, 100.3, 114.0, 115.2, 116.5, 121.7, 122.2, $123.5,124.9,125.8,128.1,132.2,134.3,140.6$, 153.4, 155.1, 157.2, 158.5, 160.0, 170.1;MS (ESI): $\mathrm{m} / \mathrm{z} 432[\mathrm{M}+1], 433[\mathrm{M}+2]$; Analysis: calcd for $\mathrm{C}_{22} \mathrm{H}_{10} \mathrm{ClN}_{3} \mathrm{O}_{3} \mathrm{~S}: \mathrm{C}, 61.19$ (61.21); H, 2.33 (2.36); N, $9.73(9.65)$.

7-(2-Chlorophenyl)-5-oxo-3-(2-oxo-2H-chromen3-yl)-5H-thiazolo[3,2-a]pyrimidine-6-carbonitrile (3c)

Pale yellow solid; m.p. $146-148^{\circ} \mathrm{C}$; yield 65; IR (KBr) $v_{\max }$ : 2226, 1676, $1712 \mathrm{~cm}^{-1}$; ${ }^{1} \mathrm{H} \mathrm{NMR}$ $\left(\mathrm{DMSO}-d_{6}, 300 \mathrm{MHz}\right) \delta \mathrm{ppm}: 6.06(\mathrm{~s}, 1 \mathrm{H}$, $\left.\mathrm{ArH}_{\text {thiazolo }}\right), 7.12$ (s, 1H, $\left.\mathrm{ArH}\right), 7.14$ (d, 1H, $J=8.4$ $\mathrm{Hz}, \mathrm{ArH})$ 7.27-7.72 (m, 7H, ArH); ${ }^{13} \mathrm{C} \mathrm{NMR}$ $\left(\mathrm{DMSO}-d_{6}, 75 \mathrm{MHz}\right) \delta \mathrm{ppm}: 94.0,102.1,112.6$, $113.1,118.7,120.1,121.8,122.0,123.1,124.6$, $126.7,133.3,134.8,142.4,149.0,156.6,156.9$, 157.3, 159.5, 168.3; MS (ESI): $m / z 432$ [M+1], 433 [M+2]; Analysis: calcd for $\mathrm{C}_{22} \mathrm{H}_{10} \mathrm{ClN}_{3} \mathrm{O}_{3} \mathrm{~S}: \mathrm{C}, 61.19$ (61.23): H, 2.33 (2.36): N, 9.73 (9.70).

7-(2,4-Dichlorophenyl)-5-oxo-3-(2-oxo-2Hchromen-3-yl)-5H-thiazolo[3,2-a]pyrimidine-6carbonitrile (3d)

Yellow solid; m.p. $116-118^{\circ} \mathrm{C}$; yield $75 \%$; IR (KBr) $v_{\max }: 2220,1656,1710 \mathrm{~cm}^{-1}$; ${ }^{1} \mathrm{H} \mathrm{NMR}$ $\left(\mathrm{DMSO}-d_{6}, 300 \mathrm{MHz}\right) \delta \mathrm{ppm}: 6.10(\mathrm{~s}, 1 \mathrm{H}$, $\left.\mathrm{ArH}_{\text {thiazolo }}\right), 6.56(\mathrm{~s}, 1 \mathrm{H}, \mathrm{ArH}), 7.02(\mathrm{t}, 2 \mathrm{H}, J=8.1$, ArH), 7.58-7.84 (m, 6H, ArH); ${ }^{13} \mathrm{C}$ NMR (DMSO- $d_{6}$, $75 \mathrm{MHz}) \delta$ ppm: 93.4, 101.1, 108.8, 112.6, 115.1, $120.3,121.6,124.1,124.7,126.2,127.3,128.1$, $129.2,130.5,132.8,133.6,138.2,152.1,158.3$, 159.3, 160.2, 170.5; MS (ESI): $m / z 468[\mathrm{M}+2]$; Analysis: Calcd for $\mathrm{C}_{22} \mathrm{H}_{9} \mathrm{Cl}_{2} \mathrm{~N}_{3} \mathrm{O}_{3} \mathrm{~S}$ : C, 56.67 (56.42); H, 1.95 (1.98); N, 9.01 (8.96).

7-(4-Fluorophenyl)-5-oxo-3-(2-oxo-2H-chromen-3yl)-5H-thiazolo[3,2-a]pyrimidine-6-carbonitrile (3e) White solid; m.p. $124-126^{\circ} \mathrm{C}$; yield $60 \%$; IR (KBr) $v_{\max }: 2236,1666,1727 \mathrm{~cm}^{-1}$; ${ }^{1} \mathrm{H}$ NMR $\left(\mathrm{DMSO}-d_{6}, 300 \mathrm{MHz}\right) \delta \mathrm{ppm}: 6.09$ (s, 1H, $\left.\mathrm{ArH}_{\text {thiazolo }}\right), 6.85-7.05$ (m, 4H, ArH), $7.58(\mathrm{~s}, 1 \mathrm{H}$, $\operatorname{ArH}), 7.77$ (t, 2H, $J=8.4 \mathrm{~Hz}, \operatorname{ArH}), 8.16(\mathrm{~d}, 2 \mathrm{H}, J$ $=8.4 \mathrm{~Hz}, \mathrm{ArH}) ;{ }^{13} \mathrm{C}$ NMR (DMSO- $\left.d_{6}, 75 \mathrm{MHz}\right) \delta$ ppm: 91.6, 102.1, 114.1, 115.5, 116.2, 118.5, 122.1, $122.9,123.2,124.1,126.0,128.8,129.0,132.3$, 153.4, 158.6, 159.4, 160.7, 162.2, 170.0; MS (ESI): m/z $415\left[\mathrm{M}^{+}\right]$; Analysis: calcd for $\mathrm{C}_{22} \mathrm{H}_{10} \mathrm{FN}_{3} \mathrm{O}_{3}: \mathrm{C}$, 63.61 (63.70); H, 2.43 (2.48); N, 10.12 (10.06).

7-(4-Nitrophenyl)-5-oxo-3-(2-oxo-2H-chromen-3yl)-5H-thiazolo[3,2-a]pyrimidine-6-carbonitrile (3f)

Brown solid; m.p. $174-176^{\circ} \mathrm{C}$; yield $80 \%$; IR (KBr) $v_{\max }$ : 2228, 1662, $1718 \mathrm{~cm}^{-1}$; ${ }^{1} \mathrm{H}$ NMR $\left(\mathrm{DMSO}-d_{6}, 300 \mathrm{MHz}\right) \delta \mathrm{ppm}: 6.00(\mathrm{~s}, 1 \mathrm{H}$, $\left.\mathrm{ArH}_{\text {thiazolo }}\right), 6.88(\mathrm{~d}, 1 \mathrm{H}, J=8.1 \mathrm{~Hz}, \mathrm{ArH}), 7.03(\mathrm{~d}$, $1 \mathrm{H}, J=7.8 \mathrm{~Hz}, \mathrm{ArH}), 7.26(\mathrm{~s}, 1 \mathrm{H}, \mathrm{ArH}), 7.29(\mathrm{t}, 2 \mathrm{H}$, $J=7.8 \mathrm{~Hz}, \mathrm{ArH}), 7.50(\mathrm{~d}, 2 \mathrm{H}, J=7.8 \mathrm{~Hz}, \mathrm{ArH}), 7.81$ $(\mathrm{d}, 2 \mathrm{H}, J=7.5 \mathrm{~Hz}, \mathrm{ArH}) ;{ }^{13} \mathrm{C}$ NMR (DMSO- $d_{6}, 75$ MHz) $\delta$ ppm: 93.2, 103.2, 116.0, 116.8, 118.6, $121.3,122.3,122.8,123.7,125.1,127.8$, $130.6,142.0,142.9,146.9,153.7,158.3,159.4$, 161.1, 169.5; MS (ESI): $m / z 443$ [M+1]; Analysis: calcd for $\mathrm{C}_{22} \mathrm{H}_{10} \mathrm{~N}_{4} \mathrm{O}_{5} \mathrm{~S}$ : C, 59.73 (59.68); H, 2.28 (2.25); N, 12.66 (12.69).

5-Oxo-3-(2-oxo-2H-chromen-3-yl)-7-(p-tolyl)-5Hthiazolo[3,2-a]pyrimidine-6-carbonitrile (3g)

Yellow solid; m.p. $142-144^{\circ} \mathrm{C}$; yield $70 \%$; IR (KBr) $v_{\max }$ : 2206, 1656, $1702 \mathrm{~cm}^{-1}$; ${ }^{1} \mathrm{H}$ NMR $\left(\mathrm{DMSO}-d_{6}, 300 \mathrm{MHz}\right) \delta \mathrm{ppm}: 2.50\left(\mathrm{~s}, 3 \mathrm{H}, \mathrm{CH}_{3}\right)$, $5.41\left(\mathrm{~s}, 1 \mathrm{H}, \mathrm{ArH}_{\text {thiazolo }}\right), 6.93(\mathrm{~d}, 2 \mathrm{H}, J=7.5 \mathrm{~Hz}$, ArH), 7.24 (d, 2H, $J=7.5 \mathrm{~Hz}, \operatorname{ArH}), 7.36$ (d, 2H, $J$ $=6.9 \mathrm{~Hz}, \operatorname{ArH}), 7.44(\mathrm{~d}, 1 \mathrm{H}, \operatorname{ArH}), 7.58(\mathrm{~s}, 1 \mathrm{H}$, ArH), 7.61 (m, 1H, ArH); ${ }^{13} \mathrm{C}$ NMR (DMSO- $d_{6} 75$ MHz) $\delta$ ppm: 21.4, 93.1, 100.1, 111.2, 114.3, 115.6, $117.3,122.3,124.1,125.7,126.1,126.8,127.4$, $128.1,135.3,141.5,151.4,155.3,158.1(\mathrm{C}=\mathrm{O})$, $159.6(\mathrm{C}=\mathrm{O}), 167.4$ (C-N);MS (ESI): $\mathrm{m} / \mathrm{z} 412$ [M+1]; Analysis: calcd for $\mathrm{C}_{23} \mathrm{H}_{13} \mathrm{~N}_{3} \mathrm{O}_{3} \mathrm{~S}$ : C, 67.14 (67.18); H, 3.18 (3.22); N, 10.21 (10.14).

5-Oxo-3-(2-oxo-2H-chromen-3-yl)-7-(o-tolyl)-5Hthiazolo[3,2-a]pyrimidine-6-carbonitrile (3h)

Brown solid; m.p. $156-158^{\circ} \mathrm{C}$; yield $65 \%$; IR (KBr) $\quad v_{\max }: \quad 2211, \quad 1660,1708 \quad \mathrm{~cm}^{-1} ; \quad{ }^{1} \mathrm{H}$ NMR(DMSO- $\left.d_{6}, 300 \mathrm{MHz}\right) \delta$ ppm: $2.49(\mathrm{~s}, 3 \mathrm{H}$, $\left.\mathrm{CH}_{3}\right), 5.99\left(\mathrm{~s}, 1 \mathrm{H}, \mathrm{ArH}_{\text {thiazolo }}\right), 6.55-7.93(\mathrm{~m}, 8 \mathrm{H}$, ArH), 8.42 (s, 1H, ArH); ${ }^{13} \mathrm{C}$ NMR (DMSO- $d_{6,75}$ MHz) $\delta$ ppm: 20.4, 92.1, 102.3, 114.5, 116.7, 117.9, $118.0,119.1,121.2,121.9,122.4,125.6,127.2$, $128.0,130.7,131.4,132.1,142.6,152.2,158.1$, 158.8, 160.3, 170.1; MS (ESI): $m / z 412$ [M+1]; Analysis: calcd for $\mathrm{C}_{23} \mathrm{H}_{13} \mathrm{~N}_{3} \mathrm{O}_{3} \mathrm{~S}$ : C, 67.14 (67.20); $\mathrm{H}, 3.18$ (3.24); N, 10.21 (10.18).

7-(4-Methoxyphenyl)-5-oxo-3-(2-oxo-2H-chromen-3-yl)-5H-thiazolo[3,2-a]pyrimidine-6-carbonitrile (3i)

Brown solid; m.p. $182-184^{\circ} \mathrm{C}$; yield $65 \%$; IR (KBr) $v_{\max }$ : 2224, 1661, $1727 \mathrm{~cm}^{-1}$; ${ }^{1} \mathrm{H}$ NMR 
(DMSO- $\left.d_{6}, 300 \mathrm{MHz}\right) \delta \mathrm{ppm}: 3.80\left(\mathrm{~s}, 3 \mathrm{H}, \mathrm{OCH}_{3}\right)$, $5.70\left(\mathrm{~s}, 1 \mathrm{H}, \mathrm{ArH}_{\text {thiazolo }}\right), 6.87(\mathrm{~d}, 2 \mathrm{H}, J=8.4 \mathrm{~Hz}$, $\operatorname{ArH}), 6.98(\mathrm{~d}, 2 \mathrm{H}, J=7.8 \mathrm{~Hz}, \operatorname{ArH}), 7.66(\mathrm{~s}, 1 \mathrm{H}$, ArH), 7.73 (m, 2H, ArH), 8.03 (d, 2H, $J=8.4 \mathrm{~Hz}$, ArH); ${ }^{13} \mathrm{C}$ NMR (DMSO- $\left.d_{6,} 75 \mathrm{MHz}\right) \delta$ ppm: 55.6, 93.2, 102.4, 114.3, 115.6, 116.4, 118.5, 125.4, $125.3,127.6,127.9,128.7,129.5,130.2,142.6$, 153.6, 158.1, 159.3, 159.6 $(\mathrm{C}=\mathrm{O}), 160.2(\mathrm{C}=\mathrm{O})$, 168.2 (C-N); MS (ESI): $m / z 428$ [M+1]; Analysis: calcd for $\mathrm{C}_{23} \mathrm{H}_{13} \mathrm{~N}_{3} \mathrm{O}_{4} \mathrm{~S}$ : C, 64.63 (64.69); H, 3.07 (3.15); N, 9.83 (9.75).

7-(2-Methoxyphenyl)-5-oxo-3-(2-oxo-2H-chromen-3-yl)-5H-thiazolo[3,2-a]pyrimidine-6-carbonitrile (3j)

Yellow solid; m.p. $168-170^{\circ} \mathrm{C}$; yield $65 \%$; IR (KBr) $v_{\max }$ : 2223, 1665, $1733 \mathrm{~cm}^{-1}$; ${ }^{1} \mathrm{H}$ NMR $\left(\mathrm{DMSO}-d_{6}, 300 \mathrm{MHz}\right) \delta \mathrm{ppm}: 3.75\left(\mathrm{~s}, 3 \mathrm{H}, \mathrm{OCH}_{3}\right)$, 5.20 (s, 1H, $\left.\operatorname{ArH}_{\text {thiazolo }}\right), 6.89$ (d, 2H, $\left.J=8.4, \mathrm{ArH}\right)$, 7.20 (s, 1H, ArH), 7.37-7.87 (m, 6H, ArH); ${ }^{13} \mathrm{C}$ NMR (DMSO- $\left.d_{6}, 75 \mathrm{MHz}\right) \delta$ ppm: 54.1, 93.2, 101.6, $112.1,114.1,115.6,118.5,118.9,120.3,120.6$, $122.1,123.2,125.8,126.1,128.9,129.0,140.1$, 150.7, 155.0, 157.2, 158.3, 159.4, 170.6; MS (ESI): $\mathrm{m} / \mathrm{z} 428$ [M+1]; Analysis: calcd for $\mathrm{C}_{23} \mathrm{H}_{13} \mathrm{~N}_{3} \mathrm{O}_{4} \mathrm{~S}: \mathrm{C}$, 64.63 (64.60); H, 3.07 (3.13); N, 9.83 (9.87).

7-(2,4-Dimethoxyphenyl)-5-oxo-3-(2-oxo-2Hchromen-3-yl)-5H-thiazolo[3,2-a]pyramid-ine-6carbonitrile $(3 \mathrm{k})$

Yellow solid; m.p. $170-172^{\circ} \mathrm{C}$; yield $85 \%$; IR (KBr) $v_{\max }$ : 2231, 1644, $1713 \mathrm{~cm}^{-1}$; ${ }^{1} \mathrm{H}$ NMR $\left(\mathrm{DMSO}-d_{6}, 300 \mathrm{MHz}\right) \delta \mathrm{ppm}: 3.76,3.86(\mathrm{~s}, 6 \mathrm{H}$, $\left.2 \mathrm{xOCH}_{3}\right), 6.12\left(\mathrm{~s}, 1 \mathrm{H}, \mathrm{ArH}_{\text {thiazolo }}\right), 7.25(\mathrm{~s}, 1 \mathrm{H}, \mathrm{ArH})$, $7.32(\mathrm{t}, 2 \mathrm{H}, J=7.8 \mathrm{~Hz}, \operatorname{ArH}), 7.41(\mathrm{~s}, 1 \mathrm{H}, \operatorname{ArH})$, 7.50 (m, 2H, ArH), 7.76 (d, 1H, $J=7.5 \mathrm{~Hz}, \operatorname{ArH})$, 7.94 (d, $1 \mathrm{H}, J=7.5 \mathrm{~Hz}, \mathrm{ArH}) ;{ }^{13} \mathrm{C}$ NMR (DMSO- $d_{6}$, $75 \mathrm{MHz}) \delta$ ppm: 55.6, 56.1, 92.6, 100.1, 111.4, $112.6,116.1,116.1,116.8,118.1,120.5,122.6$, $124.5,125.2,127.8,128.6,138.6,148.6,152.4$, 157.1, 157.9, 158.5, 158.8, 169.6. MS (ESI): $\mathrm{m} / \mathrm{z}$ $458[\mathrm{M}+1]$; Analysis: calcd for $\mathrm{C}_{24} \mathrm{H}_{15} \mathrm{~N}_{3} \mathrm{O}_{5} \mathrm{~S}$ : C, 63.01 (63.07); H, 3.31 (3.35); N, 9.19 (9.14).

7-(Naphthalen-1-yl)-5-oxo-3-(2-oxo-2H-chromen3-yl)-5H-thiazolo[3,2-a]pyrimidine-6-carbonitrile (3l)

Yellow solid; m.p. $194-196^{\circ} \mathrm{C}$; yield $75 \%$; IR (KBr) $v_{\max }: 2228,1651,1729 \mathrm{~cm}^{-1}$; ${ }^{1} \mathrm{H}$ NMR $\left(\mathrm{DMSO}_{6}, 300 \mathrm{MHz}\right) \delta \mathrm{ppm}: 5.37$ (s, $1 \mathrm{H}$, $\left.\mathrm{ArH}_{\text {thiazolo }}\right), 7.24-8.26(\mathrm{~m}, 12 \mathrm{H}, \mathrm{ArH}) ;{ }^{13} \mathrm{C} \mathrm{NMR}$ (DMSO-d 6 , 75 MHz) $\delta$ ppm: 93.9, 102.0, 115.1, $116.8,118.3,120.5,121.3,123.8,124.0,124.3$, $125.1,126.8,127.2,127.3,128.6,129.3,130.1$, $131.6,133.1,135.4,142.1,153.9,158.7,159.3$, 160.2, 170.2. m/z: 448 [M+1]; Analysis: calcd for $\mathrm{C}_{26} \mathrm{H}_{13} \mathrm{~N}_{3} \mathrm{O}_{3} \mathrm{~S}$; C, 69.79 (69.85); H, 2.93 (2.86); N, 9.39 (9.34).

\section{In vitro antimycobacterial activity}

The synthesized compounds were screened in vitro against $M t b \mathrm{H} 37 \mathrm{Rv}$ through a High Throughput Screen (HTS) using a microplate Alamar blue assay (MABA) in a 384-well plate format as reported by Collins and Franzblau (21). Handlings of $M t b$ $\mathrm{H} 37 \mathrm{Rv}$ were carried out following the protocols of Biosafety in Microbiological and Biomedical Laboratories (BMBL) 5th Edition in Biosafety

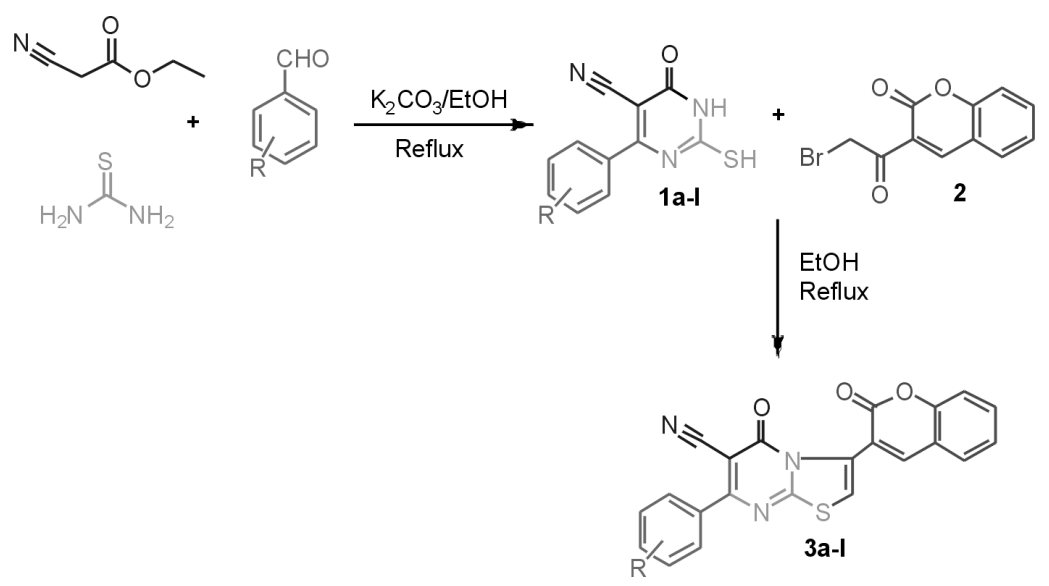

Scheme 1. Synthetic protocol for titled compounds 3a-I. 
Table 1. Antimycobacterial activity of hybrid coumarin-thiazolo[3,2-a]pyrimidine.<smiles></smiles>

\begin{tabular}{|c|c|c|c|c|}
\hline Compounds & $\mathbf{R}$ & $\mathbf{I C}_{\mathbf{5 0}}(\mathbf{\mu M})$ & $\mathbf{M I C}(\mathbf{\mu M})$ & Cytotoxicity \\
\hline $\mathbf{3 a}$ & $\mathrm{H}$ & 42.17 & 50.00 & $>6.25$ \\
\hline $\mathbf{3 b}$ & $4-\mathrm{Cl}$ & 11.07 & 50.00 & $>6.25$ \\
\hline $\mathbf{3 c}$ & $2-\mathrm{Cl}$ & 21.30 & 50.00 & $>6.25$ \\
\hline $\mathbf{3 d}$ & $2,4-\mathrm{Cl}_{2}$ & 03.19 & 12.50 & $>6.25$ \\
\hline $\mathbf{3 e}$ & $4-\mathrm{F}$ & 19.95 & 50.00 & $>6.25$ \\
\hline $\mathbf{3 f}$ & $4-\mathrm{NO}_{2}$ & 07.91 & 12.50 & $>6.25$ \\
\hline $\mathbf{3 g}$ & $4-\mathrm{CH}_{3}$ & 68.46 & 50.00 & $>6.25$ \\
\hline $\mathbf{3 h}$ & $2-\mathrm{CH}_{3}$ & 77.20 & 100.00 & $>6.25$ \\
\hline $3 \mathbf{3 i}$ & $4-\mathrm{OCH}_{3}$ & 47.20 & 100.00 & $>6.25$ \\
\hline $\mathbf{3 j}$ & $2-\mathrm{OCH}_{3}$ & 98.57 & 100.00 & $>6.25$ \\
\hline $\mathbf{3 k}$ & $2,4-\left(\mathrm{OCH}_{3}\right)_{2}$ & 28.28 & 100.00 & $>6.25$ \\
\hline $3 \mathbf{3 l}$ & $1-\mathrm{Naphthyl}$ & 32.17 & 100.00 & $>6.25$ \\
\hline Cycloserine & - & 12.47 & 25.00 & $>6.25$ \\
\hline Isoniazid & - & 0.18 & 0.31 & $>6.25$ \\
\hline Pyrimethamine & - & 37.35 & 100.00 & $>6.25$ \\
\hline
\end{tabular}

Level 3 containment laboratories. In brief, our assay uses MTB H37Rv in a 384-well plate format with in-plate DMSO carrier as growth control, two concentrations of standard anti-TB drug amikacin (3.12 $\mu \mathrm{M} \& 0.19 \mu \mathrm{M})$ as antibiotic controls and $120 \mathrm{com}-$ pounds. Hundred percent bacterial growth was observed for $M t b$ H37Rv with DMSO carrier control whereas, complete growth inhibition was observed for the $3.12 \mu \mathrm{M}$ concentration of amikacin. Amikacin at the concentration of the $0.19 \mu \mathrm{M}$ approximates the MIC of amikacin ranging from 30$80 \%$ inhibition which indicates the positive-growth inhibition and validates our assay protocols. The media used for both compound preparation and MTB H37Rv plating was assessed for contamination by plating two 384 well plates with media alone. The plates were checked for contamination by visual inspection and end-point detection. The compounds were evaluated in a 10-point stacked plate dose-response method. Compounds were serially diluted 1:2 from $100 \mu \mathrm{M}$ to $0.195 \mu \mathrm{M}$ (ten dilutions). The plates were read fluorometrically after incubation with the compounds and addition of Alamar blue.

\section{Auto-fluorescence}

The compounds in the media were pre-read from the high concentration plate with no Alamar Blue or bacteria added. The fold increase was calculated using the median of the positive control wells from the Alamar Blue $M t b \mathrm{H} 37 \mathrm{Rv}$ assay for the Mtb H37Rv control wells. For a compound to be considered autofluorescent, the criteria are defined as $>50 \%$ fluorescence of the Mtb H37Rv control wells. None of the analyzed compounds were found to be autofluorescent.

\section{Antimicrobial activity}

All the synthesized compounds $\mathbf{3 a}-\mathbf{l}$ were screened for their antibacterial activity against Staphylococcus aureus (ATCC-25923) and Escherichia coli (ATCC-25922) whereas, antifungal activity against Aspergillus niger (ATCC-9029) and Candida albicans (ATCC-90028) in DMF using serial plate dilution method at $200 \mu \mathrm{g} / \mathrm{mL}, 100$ $\mu \mathrm{g} / \mathrm{mL}, \quad 50 \mu \mathrm{g} / \mathrm{mL}, 25 \mu \mathrm{g} / \mathrm{mL}, 12.5 \mu \mathrm{g} / \mathrm{mL}, 6.25$ $\mu \mathrm{g} / \mathrm{mL}, 3.12 \mu \mathrm{g} / \mathrm{mL}, 1.56 \mu \mathrm{g} / \mathrm{mL}, 0.78 \mu \mathrm{g} / \mathrm{mL}, 0.39$ $\mu \mathrm{g} / \mathrm{mL}$ and $0.19 \mu \mathrm{g} / \mathrm{mL}$ concentrations $(22,23)$. Gentamycin and fluconazole drugs were used as reference standard for comparison (Table 2). 


\section{RESULTS AND DISCUSSION}

\section{Chemistry}

The title compounds coumarin-thiazolo[3,2a]pyrimidine (3a-l) were prepared as depicted in scheme 1 . The intermediates, 2-mercapto-6-oxo-4aryl-1,6-dihydropyrimidine-5-carbonitriles (1a-l) were synthesized by the multi-component Biginelli reaction of ethyl cyanoacetate, aryl aldehydes and thiourea, in the presence of potassium carbonate. The formation of compounds was finally confirmed by matching their physical constants. 3-(2Bromoacetyl)- $2 H$-chromen-2-one (2) was prepared by the bromination of 3 -acetylcoumarin in the presence of bromine in chloroform. Finally, the condensation of compound $\mathbf{2}$ with a different Biginelli product, mercaptopyrimidines (1a-l), resulted in coumarin-thiazolo[3,2-a]pyrimidine derivatives 3a1. The structure of the newly synthesized compounds was confirmed by IR, ${ }^{1} \mathrm{H}$ NMR and Mass spectra. The IR spectrum of compound $3 \mathbf{i}$ showed $\mathrm{C}=\mathrm{N}$ and $\mathrm{C}=\mathrm{O}$ stretching vibrations at 1661 and $1727 \mathrm{~cm}^{-1}$, respectively. The ${ }^{1} \mathrm{H}$ NMR spectral data of compound $3 \mathbf{i}$ showed a singlet at $\delta 3.80$ due to $-\mathrm{OCH}_{3}$ protons. Furthermore, the appearance of a singlet at $\delta 5.70$ due to a thiazolyl proton and the disappearance of an up-fielded signal of acetyl protons ($\mathrm{COCH}_{2}$ ) of intermediate 2 also confirms the formation of thiazolopyrimidines. The remainder of the aromatic protons were observed at $\delta 6.87-8.03 \mathrm{ppm}$.
The ${ }^{13} \mathrm{C}$ NMR spectra of compound $3 \mathbf{i}$ showed an up-fielded aliphatic peak at $\delta 55.6$ due to methoxy carbon, whereas three down-fielded peaks were observed at $\delta$ 159.6, 160.2 and 168.2 due to two $\mathrm{C}=\mathrm{O}$ and one $\mathrm{C}-\mathrm{N}$ carbons. The other seventeen peaks were observed due to aromatic carbons, thus confirming the presence of twenty-three carbons in the compound. The analytical and spectral data of all the synthesized compounds were in full agreement with the proposed structures. The elemental analysis results were within $\pm 0.4 \%$ of the theoretical values.

\section{Biological activity}

All the newly synthesized compounds 3a-l were screened by High Throughput Screen (HTS) for their in vitro antimycobacterial activity against Mtb H37Rv, using an assay adapted from the microdilution Alamar Blue (AB) broth assay. The results of the antimycobacterial screening of the twelve compounds are presented in Table 1, along with comparisons to three standard antitubercular drugs. Among the twelve newly synthesized compounds, two compounds 7-(2,4-dichlorophenyl)-5oxo-3-(2-oxo-2H-chromen-3-yl)-5H-thiazolo[3,2a]pyrimidine-6-carbonitrile (3d) and 7-(4-nitrophenyl)-5-oxo-3-(2-oxo-2H-chromen-3-yl)-5H-thiazolo[3,2-a]pyrimidine-6-carbonitrile (3f) emerged as the most active antitubercular agents, with $\mathrm{EC}_{50}$ of 3.19 and $7.91 \mu \mathrm{M}$, respectively (MICs $12.5 \mu \mathrm{M}$ ). Both of these compounds were found to be more

Table 2. In vitro antibacterial and antifungal activities of compounds 3a-I.

\begin{tabular}{|c|c|c|c|c|}
\hline \multirow{2}{*}{ Compound } & \multicolumn{2}{|c|}{ Antibacterial activity (MIC $\mu \mathrm{g} / \mathrm{mL}$ ) } & \multicolumn{2}{|c|}{ Antifungal activity (MIC $\mu \mathrm{g} / \mathrm{mL}$ ) } \\
\hline & S. aureus & E. coli & C.albicans & A. niger \\
\hline 3a & $>50$ & $>50$ & $>50$ & $>50$ \\
\hline 3b & 3.12 & 6.25 & 6.25 & 6.25 \\
\hline $3 c$ & 12.5 & 12.5 & 12.5 & 12.5 \\
\hline 3d & 0.78 & 1.56 & 1.56 & 1.56 \\
\hline $3 e$ & 12.5 & 12.5 & 25 & 25 \\
\hline $3 f$ & $>50$ & $>50$ & $>50$ & $>50$ \\
\hline $3 g$ & $>50$ & $>50$ & $>50$ & $>50$ \\
\hline $3 \mathbf{h}$ & $>50$ & $>50$ & $>50$ & $>50$ \\
\hline $3 \mathbf{i}$ & $>50$ & $>50$ & $>50$ & $>50$ \\
\hline $3 \mathbf{j}$ & $>50$ & $>50$ & $>50$ & $>50$ \\
\hline $3 \mathbf{k}$ & $>50$ & $>50$ & $>50$ & $>50$ \\
\hline 31 & $>0$ & $>50$ & $>50$ & $>50$ \\
\hline Gentamycin & 0.39 & 0.78 & - & - \\
\hline Fluconazole & - & - & 0.78 & 1.56 \\
\hline
\end{tabular}

(-) indicates not tested 
potent than the standard drugs cycloserine $\left(\mathrm{EC}_{50}\right.$ $12.47 \mu \mathrm{M})$ and pyrimethamine $\left(\mathrm{EC}_{50} 37.35 \mu \mathrm{M}\right)$, although less potent than the drug, isoniazid $\left(\mathrm{EC}_{50}\right.$ $0.18 \mu \mathrm{M})$. Five compounds $\mathbf{3 a}(\mathrm{R}=\mathrm{H}), \mathbf{3 b}(\mathrm{R}=p$ $\mathrm{Cl}), 3 \mathbf{c}(\mathrm{R}=o-\mathrm{Cl}), \mathbf{3 e}(\mathrm{R}=p-\mathrm{F})$ and $\mathbf{3 g}\left(\mathrm{R}=p-\mathrm{CH}_{3}\right)$ were found to be moderately effective (MIC $50 \mu \mathrm{M}$ ), showing $50 \%$ inhibition at concentrations of 42.17 , $11.07,21.30,19.95$ and $68.46 \mu \mathrm{M}$, respectively. The rest of the compounds $3 \mathbf{h}\left(\mathrm{R}=o-\mathrm{CH}_{3}\right), 3 \mathbf{i}(\mathrm{R}=p$ $\left.\mathrm{OCH}_{3}\right), 3 \mathbf{j}\left(\mathrm{R}=o-\mathrm{OCH}_{3}\right), 3 \mathbf{k}\left(\mathrm{R}=o, p-\left(\mathrm{OCH}_{3}\right)_{2}\right)$, and 3l $(\mathrm{R}=$ naphthyl) were found to be least effective against Mtb H37Rv (MICs $100 \mu \mathrm{M}$ ).

The general structure-activity relationship (SAR) of these hybrid coumarin analogs showed that the compounds with electron-withdrawing groups at a phenyl ring were more active than the compounds having electron-releasing groups. Also, para-substituted phenyl derivatives were more beneficial than their respective ortho-substituted derivatives, which could be due to a steric hindrance. Surprisingly, however, di-substitutions at the ortho and para positions of the phenyl ring led to an increase in antimycobacterial activity.

All the compounds were finally evaluated for cytotoxicity $\left(\mathrm{IC}_{50}\right)$ in VERO cells at higher concentrations of $62.5 \mu \mathrm{g} / \mathrm{mL}$. After three days of exposure, cellular viability was assessed based on a cellular conversion of 3-(4,5-dimethylthiazol-2-yl)2,5diphenyl tetrazolium bromide (MTT) into a formazan product using the Promega Cell Titer 96 nonradioactive cell proliferation method (17). None of the tested compounds showed any sign of cytotoxicity. Thus, the hybrid coumarin analogs were found to be safer and more effective antitubercular agents than the current compounds.

All the newly synthesized compounds 3a-1 were also evaluated for their antibacterial activity ( $S$. aureus, E. coli) and antifungal activity (C. albicans, A. niger) and compared with the standard antibiotic and antifungal drugs gentamycin and fluconazole, respectively (Table 2). Among the twelve coumarin derivatives (3a-l) evaluated, only two compounds $\mathbf{3 b}$ and 3d showed excellent antimicrobial activity (MIC $=6.25 \mu \mathrm{g} / \mathrm{mL}$ ). Compound 3b (MIC $3.12 \mu \mathrm{g} / \mathrm{mL}$ ) having dicholoro substitution at the phenyl ring was found to be the most effective antibacterial agent having MIC values 3.12-6.25 $\mu \mathrm{g} / \mathrm{mL}$.

\section{CONCLUSION}

A series of hybrid coumarin-thiazolo[3,2a]pyrimidine analogs were synthesized and evaluated for their antimycobacterial activity. The compounds, 7-(2,4-dichlorophenyl)-5-oxo-3-(2-oxo-2H-
chromen-3-yl)-5H-thiazolo[3,2-a]pyrimidine-6-carbonitrile and 7-(4-nitrophenyl)-5-oxo-3-(2-oxo-2Hchromen-3-yl)-5H-thiazolo[3,2-a]pyrimidine-6-carbonitrile represent valuable lead molecules in the exploration for antitubercular agents. Further studies regarding the quantitative structure-activity relationships (QSAR) are in progress in our laboratory.

\section{Acknowledgments}

The author (MZH) would like to express his gratitude to King Khalid University, Abha, Saudi Arabia for providing administrative and technical support.

\section{Conflict of interest}

The authors declare no conflicts of interest.

\section{REFERENCES}

1. Gagneux S.: Nat. Rev. Microbiol. 16, 202 (2018)

2. Prasad R.: Ind. J. Tuberc. 57, 180 (2010).

3. Mariandyshev A., Eliseev P.: Lancet 17, 674 (2017).

4. Adhvaryu M., Vakharia B.: Clin. Pharmacol. 3, 51 (2011).

5. WHO's Global Tuberculosis Report. 2017, WHO, Geneva.

6. Hassan M.Z., Osman H., Ali M.A., Ahsan M.J.: Eur. J. Med. Chem. 123, 236 (2016).

7. Hu Y., Shen Y., Wu X., Tu X., Wang G.X.: Eur. J. Med. Chem. 143, 958 (2018).

8. Singh A., Singh J.V., Rana A., Bhagat K., Gulati H.K. et al.: ACS Omega. 4, 11673 (2019).

9. Bhagat K., Bhagat J., Gupta M.K., Singh J.V., Gulati H.K. et al.: ACS Omega. 4, 8720 (2019).

10. Mousavi S.S., Bokharaie H., Rahimi S.: Adv. Appl. Bioinform. Chem. 3, 59 (2010).

11. Singh H., Kumar M., Nepali K., Gupta M.K., Saxena A.K. et al.: Eur. J. Med. Chem. 116, 102 (2016).

12. Singh H., Singh J.V., Gupta M.K., Saxena A.K., Sharma S. et al.: Bioorg. Med. Chem. Lett. 27, 3974 (2017).

13. Singh H., Singh J.V., Bhagat K., Gulati H.K., Sanduja M. et al.: Bioorg. Med. Chem. 27, 3477 (2019)

14. Chiang C.C., Cheng M.J., Peng C.F., Huang H.Y., Chen I.S.: Chem. Biodivers 7, 1728 (2010). 
15. Tandon R., Ponnan P., Aggarwal N., Pathak R., Baghel A.S. et al.: J. Antimicrob. Chemother. 66, 2543 (2011).

16. Cai D., Zhang Z.H., Chen Y., Yan X., Zou L. et al.: Molecules. 20, 16419 (2015).

17. Ali M.A., Sami J.G., Manogran E., Sellapan V., Hasan M.Z. et al.: Bioorg. Med. Chem Lett. 19,7000 (2009).

18. Hassan M.Z., Khan S.A., Amir M.: Eur. J. Med. Chem. 58, 206 (2012).

19. Kambe S., Saito K., Kishi H., Sakurai A., Midorikawa H.: Synthesis 4, 287 (1979).
20. Razi S.S., Srivastava P., Ali R., Gupta R.C., Dwivedi S.K. et al.: Chemicals 209, 162 (2015).

21. Collins L., Franzblau S.G.: Antimicrob. Agents Chemother. 41, 1004 (1997).

22. MacLowry J.D., Jaqua M.J., Selepak S.T.: Appl. Microbiol. 20, 46 (1970).

23. Arthington-Skaggs B.A., Motley M., Warnock D.W., Morrison C.J.: J. Clin. Microbiol. 38, 2254 (2000).

Received: 25.08 .2019 